\title{
The safe handling of bulk low-density nanomaterials
}

\author{
Varun Shenoy Gangoli ${ }^{1}$ Pavan M. V. Raja ${ }^{1}$ Gibran Liezer Esquenazi ${ }^{1}$ Andrew R. Barron ${ }^{1,2,3}$ (i)
}

(c) The Author(s) 2019 OPEN

\begin{abstract}
Nanomaterials, and in particular carbon nanotubes, possess a high surface area coupled with low density. This combination of properties renders a challenge in handling them safely, as they are susceptible to static charges, and also tend to disperse rapidly into the environment during routine handling. Their relative lightness and ease of dispersion in air also means that they cannot be handled easily in a laboratory fume hood with active air flow. One option would be to use a glove bag, but this may not always be available. Herein, we outline a safe and simple method to handle bulk amounts of low-density nanomaterials using an improvised glove box. Given the rapid rise in the manufacturing of nanomaterials, our approach may be an easy, approachable way to achieve safer working conditions-especially in the case of laboratories with constrained budgets, or start-up companies.
\end{abstract}

Keywords Low-density nanomaterials · Carbon nanotubes · SWCNTs · Worker safety

\section{Introduction}

Nanomaterials are typically defined as nanoparticles (NPs) that possess at least one dimension in the $10-100 \mathrm{~nm}$ scale. They can be of varied compositions: metal, metal oxide, organic, polymer, or a combination of two or more different materials. Despite early hype [1], the commercial adoption of nanomaterials has been slow. However, based on growing demand, it has been suggested that the nanomaterial industry will grow into a $\$ 175$ billion industry by the year 2025 [2]. One of the issues that this upsurge in applications will have to address is that nanomaterials will be synthesized and used on a larger scale than ever before. Researchers commonly study nanomaterials at the $\mu \mathrm{g}$ or mg scale; for example, a review of recent publications on carbon nanotubes (CNTs) indicates an average sample size of $10 \pm 5 \mathrm{mg}$ per experiment.
As scaling of applications occurs, academic and other research laboratories will have to handle nanomaterials at the multi-gram scale. Data discussing the extent that large scale NPs are being used in industry is limited; however, a 2008 study of 197 Swiss companies showed that NPs were being used up to $>1000 \mathrm{~kg} /$ year per company but, on average, it was more on the order of $100 \mathrm{~kg} / \mathrm{year}$ as reported by Schmid and Riediker [3]. The production of cosmetics, food, paints, powders, and the treatment of surfaces used the largest quantities of NPs.

A side effect of the small particle size and high surface area of nanomaterials is that the density of bulk materials is low. The result is nanomaterials have a light and fluffy appearance that causes air currents to disrupt their handling and result in potentially harmful exposure to researchers and workers alike. Potential routes of NP exposure include: inhalation, oral, dermal and, in the case of biomedical applications, parenteral $[4,5]$. Uptakes of NPs

\footnotetext{
Electronic supplementary material The online version of this article (https://doi.org/10.1007/s42452-019-0647-5) contains
} supplementary material, which is available to authorized users.

\footnotetext{
$\triangle$ Andrew R. Barron, arb@rice.edu; Varun Shenoy Gangoli, varunshenoyg@rice.edu; Pavan M. V. Raja, pavanraja@rice.edu|'Department of Chemistry, Rice University, Houston, TX 77005, USA. '²nergy Safety Research Institute (ESRI), Swansea University, Bay Campus, Swansea SA1 8EN, Wales, UK. ${ }^{3}$ Department of Materials Science and Nanoengineering, Rice University, Houston, TX 77005, USA.
} 
by inhalation or ingestion have been reported as the major routes for laboratory workers $[6,7]$.

Initial studies on NP hazards focused on outlining risk assessment protocols, but did not provide any practical information on the actual handling of NPs [8]. A study of safety protocols adopted by "nano-specific" facilities done by Conti et al. [9] found that fume hoods were the most widely reported engineering control; however, the highest usage was for solution manipulation, and they were less likely to be used when the NPs were a dry powder due the potential risk of exposure stemming from air turbulence generated by the fume hood exhaust system. By contrast, the performance of the air-curtain hood was consistent under all operating conditions, and any release was barely detected [10]. However, the cost of installing air-curtain hoods is relatively high, in particular for many academic institutions and start-up companies. As a reference, Hoerr et al. [11] have developed an algorithm for adopting various methods of working with NPs.

Unfortunately, adoption of sufficient safety protocols is an issue. A study by Conti et al. [9] showed that, when using NPs, there was a significant fraction of companies in both Asia and USA that did not use any form of hood system: $27.9 \%$ and $16.0 \%$, respectively. Thus, there is a significant need for a simple, flexible, and low cost system for handling large quantities of NPs, in particular 'fluffy' lowdensity materials such as carbon nanotubes (CNTs).

The goal of this work is to provide a solution to address this need, with particular relevance to academic institutions and research entities. As a low cost, easily implemented, solution, it means that no researcher or industry personnel should have an excuse in taking a shortcut to safety.

\section{Methodology}

\subsection{Materials and characterization}

Raw HiPco single-walled carbon nanotubes (SWCNTs) were obtained from Atom Nanoelectronics (Los Angeles, CA). The sample was a mixture of batches synthesized by Tubes@Rice (Rice University, Houston, TX) and used as such without further modification. The SWCNTs were characterized by SEM and Raman spectroscopy as detailed in the electronic supplementary material (ESM).

\subsection{Personal protective equipment}

The workers handling the nanomaterials should be attired as shown in Fig. 1. As required in most laboratory work, the workers have to wear closed-toed shoes, full-length trousers, and long sleeve shirts. A full-length laboratory
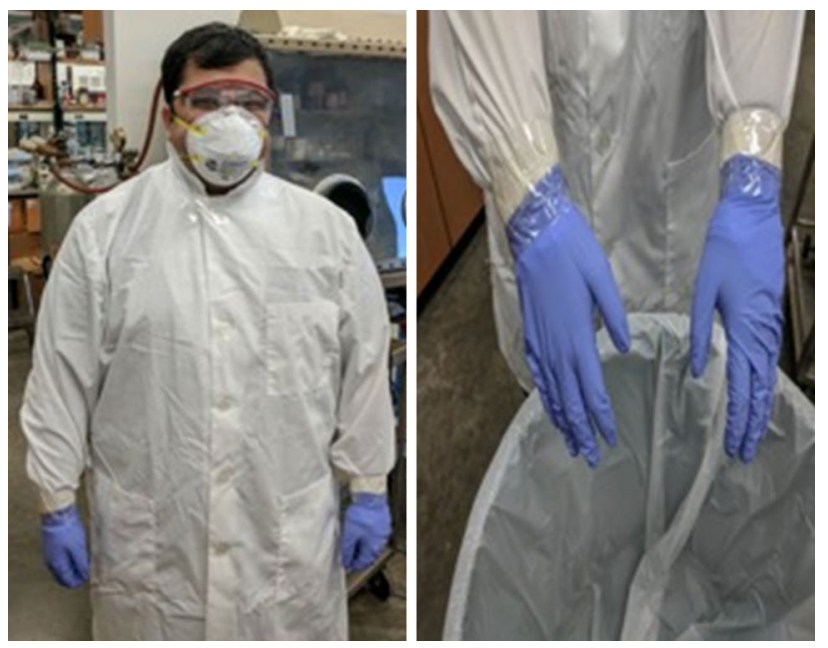

Fig. 1 Worker attire with personal protective equipment for safe handling of low-density nanomaterials such as CNTs

coat or apron is to be worn over these clothes. Two sets of blue-colored nitrile gloves are used so that there is at least one layer of redundancy in case the outer layer of gloves gets compromised during the handling process. The gloves are taped to the long sleeves of the laboratory coat using duct tape (Fig. 1b). In addition to the gloves, the collars of the aprons are drawn closed and buttoned up to the maximum extent possible such that the neck is protected. Goggles rather than safety glasses are used, as they are designed such that they surround the eyes from both sides in addition to shielding the front view from dust. Finally, a suitable face mask/shield/respirator is to be employed. These are standard equipment, that should be used when handling low-density materials, such that the eyes and nasal/oral entry passageways are protected.

\subsection{Improvised glove bag design}

Figure 2 illustrates the stepwise construction and use of the improvised glove bag used to decant SWCNTs from a large, storage, container into smaller containers.

Any suitable plastic container may be used; however, in the present case a large 25 US gallon plastic garbage bin of the type commonly used in laboratories is employed. The garbage bin was lined with a white domestic plastic garbage bag (Fig. 2a), which should be taped to the bin after first placing the unopened large storage container (Fig. 2b) inside the plastic bag lined bin (Fig. 2C). As shown in Fig. 2, the present example is a black plastic garbage bag, however, where available suitable transparent/translucent bags could be used. The present demonstration employs SWCNTs, which are one of the most difficult nanomaterials to transfer due to their fluffy appearance and ease of attracting static charge inside a traditional dry box. After 

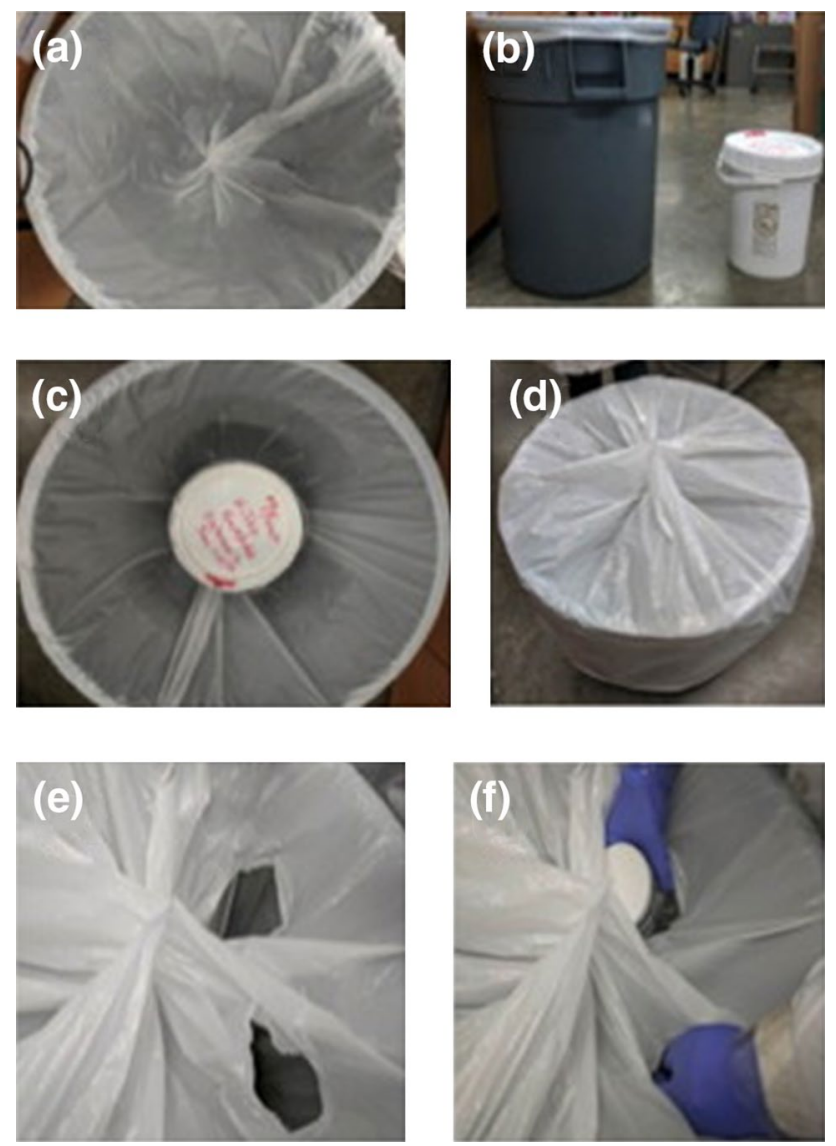

Fig. 2 Demonstration of the improvised glove bag design showing a lining of the plastic garbage container with a suitably sized plastic trash bag, the large storage container of SWCNTs, $\mathbf{b}$ beside and c inside. The second bag placed over the bin (d) with the holes cut for ingress $(\mathbf{f}, \mathbf{g})$

the large NP container is placed inside the bag-lined bin, a second plastic bag is placed over the bin opening (Fig. 2d). This second bag is to be also taped to the outside of the bin to ensure a complete seal.

Two holes were cut into the top bag cover large enough to allow the insertion of gloved hands into the glove box. The holes were also big enough to allow through the smaller containers into which the nanotubes were being transferred. However, it is important to try and make the holes as small as possible that allow easy movement without resulting in potential leakage. If any extraction container or extraction tools are bigger than the holes, place them in at the same time as the bulk NP container.

\subsection{Safe handling process}

The following represents a typical transfer of SWCNTs, but the user should repeat any steps as required. The storage container is weighed before being placed inside the $1^{\text {st }}$ plastic bag (Fig. 2c). Similarly, the aliquot containers into which the SWCNTs are to be transferred are weighed with lids in place.

Before opening the large container, the glove surfaces can be sprayed with ethanol (in the case of SWCNTs) or water, to ensure that any nanomaterial dust adheres to the glove surfaces, minimizing environmental exposure. The large container is then opened, but the lid is not removed, instead the contents are allowed to re-settle. This takes approximately $10 \mathrm{~min}$ for SWCNTs. We note that the operator is not bending during the entire process $(30-45 \mathrm{~s}$ at a time) to ensure that no strain is placed on the operator's back. If possible the entire plastic garbage bin can be raised in height as long as it is securely attached to ensure it cannot fall.

An empty aliquot container is inserted through one of the holes and the free, gloved hand was inserted through the other hole. Underneath the top cover, the lid of the aliquot container is loosened using both hands and placed on one side of the garbage bin along with the now-open aliquot container. Both hands were then used to carefully lift the lid of the bulk container upward and held at an angle over the large container by one hand, such that there is an opening large enough to allow the extraction aliquot container to pass through and into the bulk container. This needs to be done carefully and deliberately such that mechanical disturbances are minimized. The lid of the large container is used to scrape any excess back into storage container. The aliquot container, having being dipped into the bulk container, is to be slowly withdrawn such that it is now filled with the SWCNTs, following which the lid of the bulk container was lowered back (but not sealed, see below), and the aliquot container is quickly capped with its lid using the free hand.

As one of the gloved hands lifted the filled and capped aliquot container out of the trash can, another worker stands by with paper wipes soaked in acetone so that they could wipe off the gloved hands and the aliquot container surface as they emerge from the trash can. This is so that the acetone can soak the nanomaterials and make them less susceptible to dispersion. The container is then wiped thoroughly using more solvent-soaked paper towels that should then suitably be discarded as solid waste. The cleaned container was labeled as "Aliquot 1 " in this instance, and kept aside for subsequent analysis and research. The process was repeated till three other similar aliquot containers were filled up.

Once sufficient nanomaterials are transferred the lid of the storage container is sealed in place. The outside of the storage container is wiped with EtOH-wetted paper towel. Next, a spray bottle of acetone is taken into the bag and used to spray the inside of the trash bag and outside of the large bulk container. Using fresh towels, the container is cleaned, and the gloves are replaced. 
Following the aliquot preparation process, the garbage bag lid of the trash bin was detached by removing the tape holding it and the underside was liberally sprayed with acetone to minimize dispersion of nanomaterial dust. Then, the bulk container was wiped while still in the garbage bag lined trash bin using solvent-soaked tissue wipes, such that its surface was clean again before removing it from the trash container.

Once the storage container is removed, more solvent was added to the can so that any remaining nanomaterial dust could settle, aggregate, and adhere to the lining material of the garbage bag. The trash bag lining was carefully removed and further sprayed with acetone, before folding them up and disposing as solid waste. The first trash bag is then removed from inside the garbage bin and tied with all the paper towels and both bags, along with any used towels, are disposed of as solid chemical waste. Finally, the storage container is re-weighed along with the aliquot containers, and the difference represents the waste during the transfer, which should be small.

Since excess solvent was used, and because a clean trash bag was used to start with, most of the nanotubes were dispersed into the solvent and collected into another container for recovery. This meant that wastage of the expensive nanomaterials was also minimized. The garbage bin was now made ready for use again after lining it with a new trash bag.

\section{Results and discussion}

\subsection{Personal protective equipment}

In considering a method for transferring fluffy NPs such as SWCNTs it is necessary to understand what routes of exposure must be mitigated. Table 1 mentions some of the possible routes of worker exposure to nanomaterials, as well as prevalent safe working practices/protective equipment. However, these methods primarily relate to handling of miniscule quantities of materials and would most likely need improvisation when the respective processes need to be scaled up.

As has been noted before, performance of the aircurtain hood has been found to be ideal for the handling of NPs [10]; however, the cost of installation is high and requires space and infrastructure. Thus, a simpler, more easily adopted protocol must be developed that ensures safe handling of NPs, in particular for many academic institutions.

The use of respirators is often suggested when engineering controls do not adequately keep worker exposure to an airborne contaminant below a regulatory limit or internal target $[12,13]$. Unfortunately, there remain uncertainties as to whether certified respirators offer the needed level of protection against nanoparticles [14]. Irrespectively, facial protection was also employed using suitable face masks and safety goggles. The choice of face masks is again dictated by the nature of the experiment or process. Respirators may be necessary if toxic volatile compounds or solvents are involved. The goggles should be designed such that they surround the eyes from both sides, in addition to shielding the front view from dust. While a cap is not shown in Fig. 1, it should also be used by workers with long hair or when handling even larger quantities of particulate materials.

Standard tests showed that non-woven fabrics, like high-density polyethylene textile (Tyvek ${ }^{\circ}$ type), seems to be much more efficient against nanoparticles penetrations. Thus, it is advised to avoid the use of protective clothing made with cotton fabrics $[15,16]$. New nanofibrous mats of polyamide- 6 have been tested as alternative protective clothing for NP exposure [17]. It is also the policy in our laboratories to dispose of used aprons periodically such that contaminants are not allowed to accumulate on the aprons over time.

Surprisingly, it has been found that in many instances no gloves are used when handling NPs: Asia 32.6\%, Europe $18.2 \%$, and USA $12.0 \%[9,14]$. This is especially troubling since the transdermal transport of NPs has been demonstrated [5]. Table 2 provides the designed applications for various gloves based on the Berkeley Glove Selection Guide [18]. In most cases, nitrile rubber gloves are suitable for handling NPs, and particularly allow for being washed with organic solvents used to aggregate the NPs or during clean up. For example, certain gloves cannot be used when non-polar solvents or acids are involved. In the current study, blue-colored nitrile gloves were used. The glove color also helps provide a contrast to the color of the material being handled such that exposure to nanomaterials can be identified easily. Double gloves were used so

Table 1 Possible exposure routes and remedial measures pertaining to nanomaterials

\begin{tabular}{lll}
\hline Routes to exposure & Effects & Mitigation \\
\hline Skin, eyes, nose, mouth & $\begin{array}{l}\text { Dermatitis, eye damage, respiratory Illness, autoim- } \\
\text { mune diseases, cell damage, cardiac and circulatory } \\
\text { problems }\end{array}$ & $\begin{array}{c}\text { Gloves, hood/glove box, safety glasses/face shield/face } \\
\text { mask, respirator, lab coat/apron }\end{array}$ \\
\hline
\end{tabular}


Table 2 Types of gloves used for various applications [18]

\begin{tabular}{|c|c|c|c|}
\hline Type & Pros & Cons & Applications \\
\hline Latex & Compatible with aqueous solutions & $\begin{array}{l}\text { Latex allergies; unsuitable with } \\
\text { organic solvents }\end{array}$ & $\begin{array}{l}\text { Protection against aqueous chemicals, } \\
\text { where latex allergies are not involved }\end{array}$ \\
\hline Nitrile & $\begin{array}{l}\text { Compatible with a good range of } \\
\text { chemicals, no allergies caused }\end{array}$ & $\begin{array}{l}\text { May need thicker gloves for extended } \\
\text { contact with harsh chemicals }\end{array}$ & $\begin{array}{l}\text { For general purpose sample examina- } \\
\text { tion }\end{array}$ \\
\hline Butyl Rubber & Compatible with esters and ketones & Unsuitable for handling hydrocarbons & $\begin{array}{l}\text { Extended contact with selected chemi- } \\
\text { cals/solvent }\end{array}$ \\
\hline Neoprene & $\begin{array}{l}\text { Compatible with alkalis, acids, many } \\
\text { hydrocarbons, etc. }\end{array}$ & $\begin{array}{l}\text { Unsuitable for handling modified } \\
\text { hydrocarbons (e.g., aromatic/halo- } \\
\text { genated) }\end{array}$ & $\begin{array}{l}\text { Extended contact with hazardous } \\
\text { chemicals unless contra-indicated }\end{array}$ \\
\hline Polyvinyl Chloride & $\begin{array}{l}\text { Compatible with alkalis, acids, } \\
\text { fats, oils, amines, and peroxides. } \\
\text { Abrasion-resistant }\end{array}$ & $\begin{array}{l}\text { Unsuitable for handling organic } \\
\text { solvents }\end{array}$ & Specific use with compatible chemicals \\
\hline Polyvinyl Alcohol & $\begin{array}{l}\text { Compatible with chlorinated and } \\
\text { aromatic reagents }\end{array}$ & $\begin{array}{l}\text { Unsuitable for handling aqueous } \\
\text { solutions }\end{array}$ & Specific use with compatible chemicals \\
\hline Kevlar & $\begin{array}{l}\text { Compatible with cut-resistant applica- } \\
\text { tions }\end{array}$ & $\begin{array}{l}\text { Susceptible to degrade under UV } \\
\text { radiation }\end{array}$ & Handling sharp objects \\
\hline
\end{tabular}

that there was at least one layer of redundancy in case the outer layer of gloves was compromised during the handling process.

\subsection{Handling technique}

The choice of a 25 US gallon style trash bin was because it is low cost, making it economic to be disposed of if necessary, as well being freely available. In a similar manner, use of household garbage bags is intended to allow for their one-time use. Most garbage bags are flexible lowdensity polyethylene (LDPE) or, for strength, linear lowdensity polyethylene (LLDPE) or high-density polyethylene (HDPE); however, oxo-biodegradable plastic bags have the same strength as ordinary plastic and cost very little extra. The choice of bags is in part also dictated by the color of the NPs being transferred since, for example, white trash bags allows ease of visualization of the black SWCNTs.

One must bear in mind that, especially in the case of SWCNTs, nanomaterials tends to be extremely fluffy and liable to be dispersed at the slightest application of mechanical force, or in the presence of static or even a light breeze. Thus, slow, deliberate movements are preferential such that mechanical disturbances were minimized.

\subsection{Resulting nanomaterial aliquot characterization}

The handling technique employed should be such that the resulting aliquots should ideally be representative in characteristics. As an example, the SWCNT samples transferred using the techniques discussed herein four aliquots were characterized by SEM, Raman spectroscopy, and EDS analysis.
Figure S1 (see ESM) shows one of the aliquot bottles containing the transferred nanomaterial, after thoroughly wiping its external surfaces with acetone and labeling for identification. The bottles were weighed before and after the transfer process, and this allowed for calculation of the amount of material extracted in each bottle. The four aliquots had nanomaterials with masses ranging between 1.00 and $1.26 \mathrm{~g}$. The containers each had a volume of $1 \mathrm{~L}$, for reference.

SEM images of the four aliquots in Fig. S2 (see ESM) show similar morphology, while the corresponding EDS data (Fig. 3) taken from 4 randomly chosen areas of each aliquot, revealed compositions of various elements $(C, O$, and $\mathrm{Fe}$ ) to have greater variation within the sample than between aliquots. This is consistent with the observation that raw SWCNTs in general are highly heterogeneous in nature and can vary in terms of composition (due to variations in catalyst content), bundling, aggregation, and

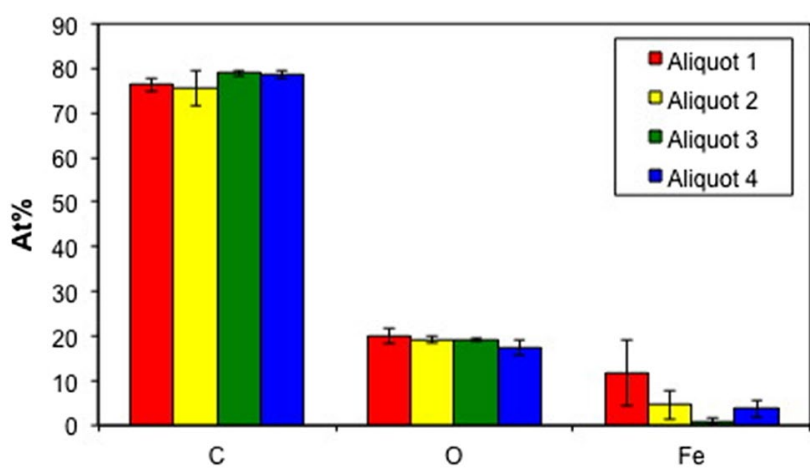

Fig. 3 Average EDS analysis of 4 aliquots taken from a large batch of SWCNTs. Each EDS analysis was taken of 4 areas giving the standard deviation showed 
defect sites [19, 20]. Figure S3 (see ESM) shows representative Raman spectra $(633 \mathrm{~nm})$ for the four aliquots. The $I_{G}: I_{D}$ ratio was found to be $14.7 \pm 0.8$ across all the samples, again suggesting consistency between the aliquots. The variations observed across the batches suggesting uniform sampling based on the chosen aliquot procedure.

\section{Conclusions}

In our study, we demonstrated an effective improvised glove bag system based on commonly available laboratory supplies and PPE that could be used to minimize unintended dispersion of low-density particulate matter, while enabling the workers to safely handle the material. Although our study focused on raw SWCNTs as a model as a low-density material, we anticipate that this technique can be used, with suitable adaptations (to meet regulatory requirements and/or in-house safety policies), to handle any other type of particulate material. The improvised glove bag technique can be used in a wide range of environments, such as academic research laboratories, R\&D laboratories of industries, start-up facilities, and largescale corporate manufacturing facilities. Furthermore, this method can potentially serve as a guideline to promote safe working practices and regulations such that occupational hazards in the nanotechnology industry can be addressed and minimized.

Acknowledgements Funding for this work was provided by the Robert A Welch Foundation (C-0002), the Office of Naval Research (N00014-15-2717), and the Welsh Government through the Sêr Cymru Chair Program. The authors would also like to thank Lt. Derek Barbee for assistance with photographs.

\section{Compliance with ethical standards}

Conflict of interest The authors declare they have no conflict of interest.

Open Access This article is distributed under the terms of the Creative Commons Attribution 4.0 International License (http://creativeco mmons.org/licenses/by/4.0/), which permits unrestricted use, distribution, and reproduction in any medium, provided you give appropriate credit to the original author(s) and the source, provide a link to the Creative Commons license, and indicate if changes were made.

\section{References}

1. Berube DM (2005) Nano-hype: the truth behind the nanotechnology buzz. Prometheus Books, Amherst

2. Global Nanotechnology Market Analysis \& Trends - Industry Forecast to 2025 (2018) Research and Markets. ID: 4699610

3. Schmid K, Riediker M (2008) Use of nanoparticles in Swiss industry: a targeted survey. Environ Sci Technol 42:2253-2260
4. Stern ST, McNeil SE (2008) Nanotechnology safety concerns revisited. Toxicol Sci 101:4-21

5. Rouse JG, Ryman-Rasmussen JP, Yang J, Barron AR, MonteiroRiviere NA (2007) Effects of mechanical flexion on the penetration of fullerene amino acid-derivatized peptide nanoparticles through skin. Nano Lett 7:155-160

6. U.S. National Institute for Occupational Safety and Health, Centers for Disease Control and Prevention (2006) Approaches to safe nanotechnology - an information exchange with $\mathrm{NIOSH}$. https://ethics.iit.edu/NanoEthicsBank/node/704

7. European Commission, Health \& Consumer Protection Directorate, Scientific Committee on Emerging and Newly Identified Health Risks (SCENIHR) (2006) The appropriateness of existing methodologies to assess the potential risks associated with engineered and adventitious products of nanotechnologies. https://ethics.iit.edu/NanoEthicsBank/node/1491

8. Maynard AD, Aitken RJ, Butz T, Colvin V, Donaldson K, Oberdörster G, Philbert MA, Ryan J, Seaton A, Stone V, Tinkle SS, Tran L, Walker NJ, Warheit DB (2006) Safe handling of nanotechnology. Nature 444:267-269

9. Conti JA, Killpack K, Gerritzen G, Huang L, Mircheva M, Delmas M, Harthorn BH, Appelbaum RP, Holden PA (2008) Health and safety practices in the nanomaterial workspace: results from an international survey. Environ Sci Technol 42:3155-3162

10. Tsai SJ, Huang RF, Ellenbecker MJ (2010) Airborne nanoparticle exposure while using constant-flow, constant-velocity, and aircurtain-isolated fume hoods. Ann Occup Hyg 54:78-87

11. Hoerr RA, Gupta A, Matuszewski MJ (2009) Developing practices for safe handling of nanoparticles and nanomaterials in a development-stage enterprise: a practical guide for research and development organizations. In: Webster T (ed) Safety of nanoparticles. Nanostructure science and technology. Springer, New York

12. Shaffer RE, Rengasamy S (2009) Respiratory protection against airborne nanoparticles: a review. J Nanopart Res 11:1661

13. Dhawan A, Shanker R, Das M, Gupta KC (2011) Guidance for safe handling of nanomaterials. J Biomed Nanotechnol 7:218-224

14. Dolez PI, Bodila N, Lara J, Truchon G (2010) Personal protective equipment against nanoparticles. Int J Nanotechnol 7:99-117

15. Ostiguy C, Lapointe G, Ménard L, Cloutier Y, Trottier M, Boutin M, Antoun M, Normand C (2006) Nanoparticles-actual knowledge about occupational health and safety risks and prevention measures. IRSST, report-470, Montréal

16. Luana G, Arnaud G, Francois T (2008) Efficiency of fibrous filters and personal protective equipments against nanoaerosols. Nanosafe, dissemination report, DR-325/326-200801-1

17. Faccini M, Vaquero C, Amantia D (2012) Development of protective clothing against nanoparticle based on electrospun fibers. J Nanomater 2012:892894

18. Berkeley EH\&S Glove Selection Guide. https://ehs.berkeley.edu/ workplace-safety/glove-selection-guide. Accessed Jan 2019

19. Pham D, Zhang KS, Lawal O, Ghosh S, Gangoli VS, Ainscough TJ, Kellogg B, Hauge RH, Adams WW, Barron AR (2017) Apparatus for scalable functionalization of single-walled carbon nanotubes via the Billups-Birch reduction. C 3:19

20. Zhang KS, Pham D, Lawal O, Ghosh S, Gangoli VS, Smalley P, Kennedy K, Brinson BE, Billups WE, Hauge RH, Adams WW, Barron AR (2017) Overcoming catalyst residue inhibition of the functionalization of single-walled carbon nanotubes via the Billups-Birch reduction. ACS Appl Mater Interfaces 9:37972-37980

Publisher's Note Springer Nature remains neutral with regard to jurisdictional claims in published maps and institutional affiliations. 